\title{
Rat limbal niche cells can induce transdifferentiation of oral mucosal epithelial cells into corneal epithelial-like cells in vitro
}

\author{
Xin-Yue Zhao' ${ }^{1}$ Hua-Tao Xie ${ }^{1}$, Chao-Ye Duan ${ }^{1}$, Jing Li ${ }^{2}$ and Ming-Chang Zhang ${ }^{1 *}$
}

\begin{abstract}
Background: Cultivated oral mucosal epithelial cells (OMECs) are widely used in the treatment of limbal stem cell deficiency (LSCD) for their ocular reconstruction capability. As the most important component of the limbal microenvironment, limbal niche cells (LNCs) play a key role in the direction of stem cell differentiation. In this study, we investigated whether LNCs can induce the transdifferentiation of rat OMECs to corneal epithelial-like cells.

Methods: We isolated OMECs and LNCs from rats by dispase and collagenase, respectively, to establish a threedimensional or Transwell coculturing system. NIH-3T3 cells and renewed LNCs were also used as feeder layers in the Transwell system to compare their ability to support the OMECs. The airlift method was used for the culture of OMECs to obtain a stratified epithelial sheet. Cocultured OMECs were characterized by reverse-transcription polymerase chain reaction, Western blotting, hematoxylin and eosin staining, and immunohistochemistry.

Results: The cocultured OMECs showed corneal epithelial-like morphology and expressed the corneal epithelial markers CK12 and Pax6 in most cocultured systems. Furthermore, we found that the expression level of CK12, Pax6, and proliferation marker Ki67 was upregulated when compared with that of other groups by renewing the LNCs in the Transwell system $(p<0.05, n=3)$, suggesting that this might be a potential method for improving the efficiency of transdifferentiation. The obtained stratified epithelial sheet expressed CK3 and CK12.
\end{abstract}

Conclusion: Through coculturing OMECs and LNCs in vitro, we successfully cultivated corneal epithelial-like OMECs. This investigation is of great significance for the treatment of LSCD and ocular surface reconstruction.

Keywords: Limbal niche cells, Oral mucosal epithelial cells, Cell transdifferentiation, Limbal stem cell deficiency, Ocular reconstruction

\section{Background}

Limbal stem cell deficiency (LSCD) is the main cause of most blinding keratopathies [1]. Autologous limbal stem cell transplantation is important for ocular reconstruction $[2,3]$, and the results of long-term follow-up are very encouraging [4]. However, the use of this method poses a threat to the healthy eye [5], and most serious ocular diseases are binocular, forcing ophthalmologists

\footnotetext{
* Correspondence: mingchangzhang@hotmail.com

${ }^{1}$ Department of Ophthalmology, Union Hospital, Tongji Medical College, Huazhong University of Science and Technology, Wuhan 430022, China Full list of author information is available at the end of the article
}

to select cornea or stem cells of allogeneic origin [6]. Nonetheless, the severe lack of corneal donors in China and the risk of rejection after transplantation restricts allogeneic transplant $[7,8]$. Nowadays, cultivated oral mucosal epithelial cell (OMEC) transplantation has shown encouraging results in reconstructing the ocular surface affected by LSCD [9-12]. NIH-3T3 cells are widely used to coculture the oral epithelium for maintenance of undifferentiated epithelial stem cells during amplification $[9,10,12]$. However, this poses a risk of transmitting murine-derived diseases. Moreover, long-term outcomes are less satisfactory; specifically, a high rate of peripheral corneal neovascularization has 
been observed [13]. Therefore, in recent years, scholars have used other fibroblasts to expand oral mucosal epithelium and corneal epithelium in vitro [11, 14].

Some researchers have reported successful application of the limbal microenvironment in vitro to stimulate embryonic stem cells and adult stem cells to transdifferentiate into corneal-like epithelium [15-19]. As the most important component of the limbal microenvironment, limbal niche cells (LNCs) play a key role in the prevention of differentiation of limbal stem cells [20-22]. Oral epithelial cells and corneal epithelial cells originate from the same ectoderm; however, the only study that attempted to coculture OMECs and LNCs in vitro obtained unsatisfactory results [23]. In this study, we cocultured OMECs and LNCs with a new in-vitro model to observe whether LNCs can induce the transdifferentiation of rat OMECs to corneal epithelial-like cells.

\section{Methods}

\section{Animals}

Sprague-Dawley rats weighing 150-200 g each were supplied by the Animal Research Committee of the Huazhong University of Science and Technology (Wuhan, China). The study was conducted after obtaining approval from the Institutional Animal Care and Use Committee at Tongji Medical College, Huazhong University of Science and Technology (IACUC number S760, 9 September 2016; Additional file 1).

\section{Isolation of rat central cornea, limbus, and OMECs}

Isolation of rat central cornea was conducted with a protocol we have reported previously [21]. Briefly, after anesthesia and dislocation of the spinal column in the rats, the eyeball was removed, followed by ring-cutting of the cornea at $1 \mathrm{~mm}$ outside the limbus of the cornea. A corneal trephine of $3 \mathrm{~mm}$ diameter was used to separate the central cornea and limbus. A portion of the tissue was embedded in paraffin for immunofluorescence, while the other portion was washed three times with phosphate-buffered saline (PBS; Hyclone, Logan, UT, USA), cut into small pieces followed by removal of the iris and endothelium, and digested with $10 \mathrm{mg} / \mathrm{mL}$ dispase II (Roche, Indianapolis, IN, USA) for $30 \mathrm{~min}$. Both central and limbal epithelium were separated from the corneal stroma by ophthalmic micro-tweezers. Isolation of OMECs was performed using a previously reported method [24]. In brief, the buccal mucosa was removed for the experiment; a portion was embedded in paraffin for immunofluorescence, while the rest was cut into small pieces and washed three times with PBS. The samples were then digested in $10 \mathrm{mg} / \mathrm{mL}$ dispase II for 30 min. OMECs were also separated by ophthalmic micro-tweezers. A flow chart outlining the steps involved in isolation is presented as Fig. 1a.

\section{Preparation of Matrigel}

The Matrigel $^{\mathrm{lm}}$ Basement Membrane Matrix (BD Biosciences, San Jose, CA, USA) was prepared in coating or three-dimensional (3D) form following previous reports [20]. Briefly, Matrigel was diluted at a ratio of 1:20 or 1:1 with Dulbecco's modified Eagle's medium (DMEM/F12, [1:1]; Hyclone) for coating or 3D, respectively. The diluted Matrigel was then added into the culture plate at $50 \mu \mathrm{L} / \mathrm{cm}^{2}$ or $200 \mu \mathrm{L} / \mathrm{cm}^{2}$ for coating or $3 \mathrm{D}$ culture, respectively, followed by incubation at $37{ }^{\circ} \mathrm{C}$ with $5 \% \mathrm{CO}_{2}$ for $1 \mathrm{~h}$. Before cell seeding, the Matrigel coating was removed. All operations were performed in an ice bath.

\section{Culturing of LNCs}

LNC culture was conducted as described previously [20], the steps of which are also included in Fig. 1a. Briefly, the limbus was washed with PBS three times and digested in $1 \mathrm{mg} / \mathrm{mL}$ Collagenase A (Sigma-Aldrich, St. Louis, MO, USA) for $3 \mathrm{~h}$. The outcome of digestion was then transferred to an Eppendorf tube for trypsinization in $0.25 \%$ trypsin $/ 0.02 \%$ ethylenediaminetetraacetic acid (T/E) for $10 \mathrm{~min}$. After neutralization, the samples were centrifuged and resuspended in modified embryonic stem cell medium (MESCM), which consisted of DMEM/F12 supplemented with $10 \%$ knockout serum, $5 \mu \mathrm{g} / \mathrm{mL}$ insulin, $5 \mu \mathrm{g} / \mathrm{mL}$ transferrin, $5 \mathrm{ng} / \mathrm{mL}$ sodium selenite, $4 \mathrm{ng} / \mathrm{mL}$ basic fibroblast growth factor, $10 \mathrm{ng} / \mathrm{mL}$ human leukemia inhibitory factor, $50 \mu \mathrm{g} / \mathrm{mL}$ gentamicin, and $1.25 \mu \mathrm{g} / \mathrm{mL}$ amphotericin B. The cells were cultured in six-well plastic culture plates coated with 5\% Matrigel. Once confluent, they were passaged at a ratio of 1:4 until passage 3 . In our studies, we also used DMEM/F12 supplemented with 10\% fetal bovine serum to culture LNCs. The obtained LNCs in MESCM or DMEM/F12 supplemented with $10 \%$ fetal bovine serum were designated ME-LNCs or DF-LNCs. The expression level of both the mesenchymal stem cell marker Sox 2 and Oct4 of P3 LNCs were examined by reverse-transcription polymerase chain reaction (RT-PCR) and Western blotting, and N-cadherin was examined by immunofluorescence. Double immunofluorescence of Vimentin (Vim) and CK12, $\Delta \mathrm{Np} 63 \alpha$, or Pax6 was also evaluated to verify that purified LNCs were obtained.

\section{Three-dimensional coculturing of OMECs and LNCs under different conditions}

Three-dimensional coculturing of OMECs and LNCs was conducted as shown in Fig. 1b. Briefly, the isolated oral mucosal epithelium was trypsinized by $\mathrm{T} / \mathrm{E}$ for $15 \mathrm{~min}$. Afterwards, the samples were neutralized, followed by centrifugation and resuspension in MESCM or supplemented hormonal epithelial medium (SHEM), which consisted of DMEM/F12 (1:1) supplemented with 10\% fetal bovine serum, $5 \mu \mathrm{g} / \mathrm{mL}$ insulin, $5 \mu \mathrm{g} / \mathrm{mL}$ transferrin, $5 \mathrm{ng} / \mathrm{mL}$ sodium selenite, $0.45 \mu \mathrm{g} / \mathrm{mL}$ hydrocortisone, $20 \mathrm{ng} / \mathrm{mL}$ 


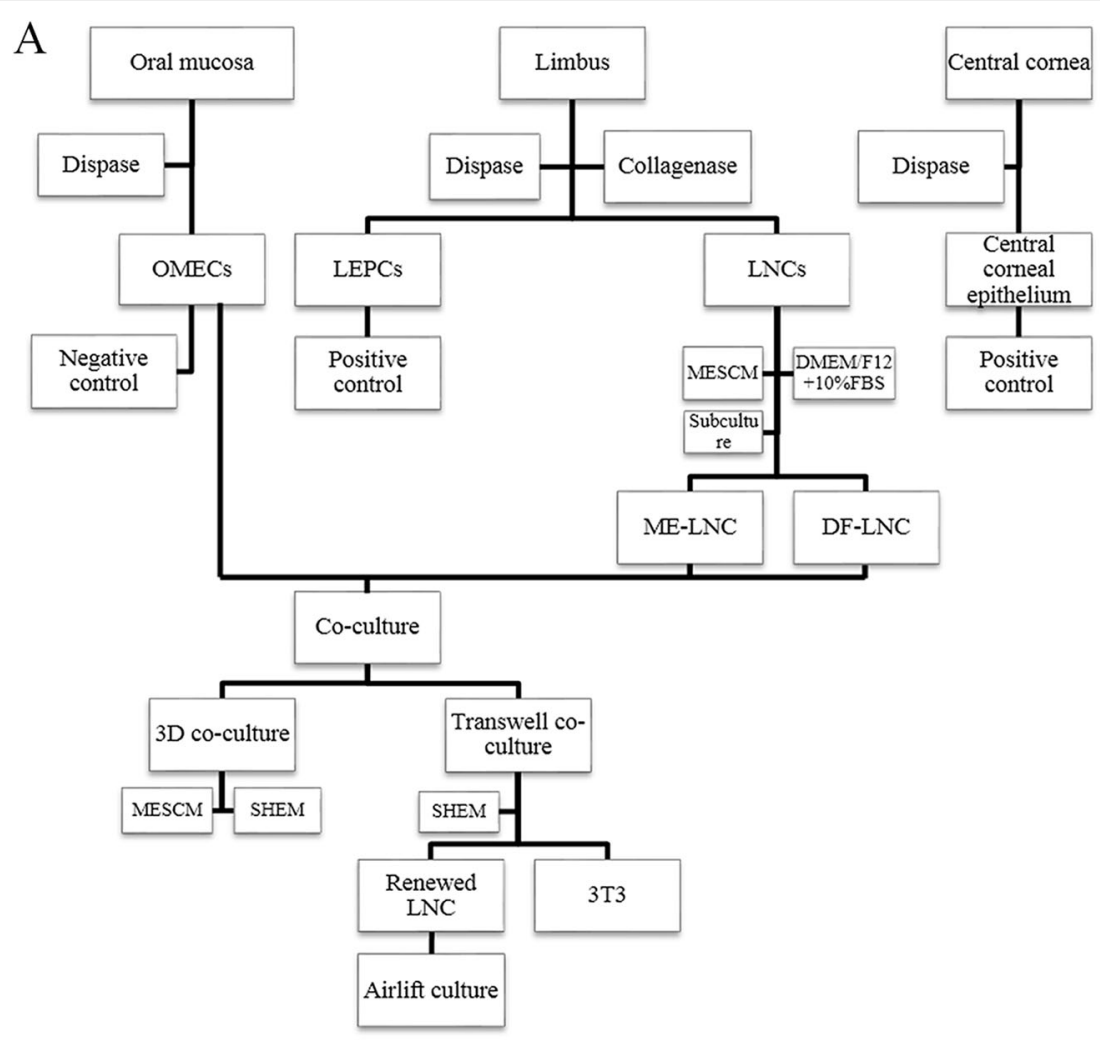

$\mathrm{B}$

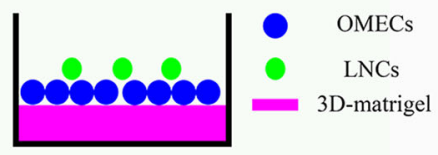

$\mathrm{D}$

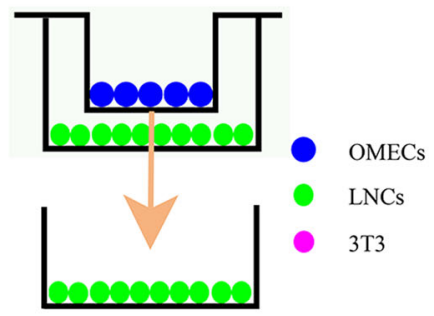

$\mathrm{C}$

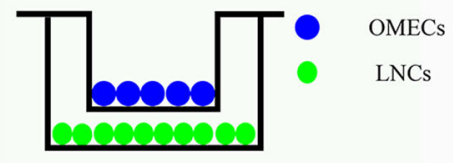

E

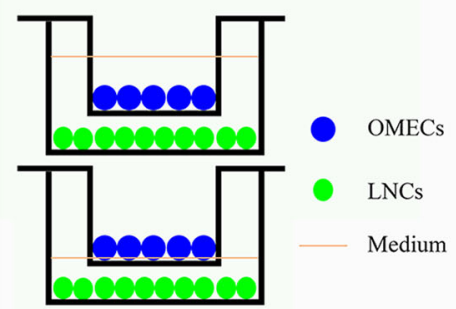

Fig. 1 Isolation and coculturing of OMECs and LNCs. a Isolation of central corneal epithelial cells, limbal epithelial progenitor cells, limbal niche cells (LNCS), and oral mucosal epithelial cells (OMECs). b Three-dimensional coculturing system, c Transwell coculturing system, $\mathbf{d}$ renewed Transwell coculturing system, and e Airlift coculturing system. DF-LNC LNCs grown in DMEM/F12 supplemented with 10\% FBS, DMEM/F12 Dulbecco's modified Eagle's medium, FBS fetal bovine serum, LEPC limbal epithelial progenitor cell, ME-LNCs LNCs grown in MESCM, MESCM modified embryonic stem cell medium, SHEM supplemented hormonal epithelial medium 
epidermal growth factor, $10 \mathrm{ng} / \mathrm{mL}$ human leukemia inhibitory factor, and $100 \mathrm{U} / \mathrm{mL}$ penicillin and streptomycin. Cell counting confirmed that the OMECs and P3 LNCs were mixed at a ratio of 4:1. Then, the mixture was seeded at a density of $12 \times 10^{4}$ cells $/ \mathrm{cm}^{2}$ for OMECs and $4 \times 10^{4}$ cells $/ \mathrm{cm}^{2}$ for LNCs in 24-well plastic culture dishes that contained 3D Matrigel. According to LNCs and the media used in the 3D coculturing system, we established four different experimental groups, including ME-ME (ME-LNCs, MESCM for 3D coculturing), ME-SH (ME-LNCs, SHEM for 3D coculturing), DF-ME (DF-LNCs, MESCM for 3D coculturing), and DF-SH (DF-LNCs, SHEM for 3D coculturing). Furthermore, the primary OMECs and central corneal epithelium were considered as controls. The culture medium was changed every 2 days. The expression level of the specific corneal markers CK12 and Pax6 in the cocultured OMECs and LNCs was observed. On day 7 of coculturing, $10 \mathrm{mg} / \mathrm{mL}$ dispase II was added to each group to dissolve the Matrigel at $37{ }^{\circ} \mathrm{C}$ with $5 \% \mathrm{CO}_{2}$ for $2 \mathrm{~h}$. A portion of the resulting samples was prepared for RT-PCR. The rest were used for immunofluorescence after trypsinization to suspend the cells. The positive rates of CK12 and Pax6 expression were determined by counting in three randomly selected 400-fold fields of vision and calculating the average positive rate; the experiment was repeated three times.

\section{Transwell coculturing of OMECs and LNCS}

We also attempted to coculture OMECs and LNCs in a Transwell system to obtain a transplantable epithelial sheet using the model provided in Fig. 1c. When the P3 ME-LNCs and DF-LNCs reached confluence, they were treated with $4 \mu \mathrm{g} / \mathrm{mL}$ mitomycin $\mathrm{C}$ for $2 \mathrm{~h}$ to restrain their growth. After washing with PBS three times, they were ready for coculturing. The Transwell inserts $(0.4 \mu \mathrm{m}$, polyethylene terephthalate; Corning Inc., Corning, NY, USA) coated with $5 \%$ Matrigel were placed in the wells. The suspended OMECs were seeded at a density of $5 \times 10^{4} / \mathrm{cm}^{2}$ in the insert. SHEM ( $1.5 \mathrm{~mL}$ and $2.6 \mathrm{~mL}$ ) was added to the upper and lower chamber, respectively, according to the Transwell instructions. According to the medium used for LNC culture in the Transwell system, we established two experimental groups, including ME (Cocultured OMECs supported by ME-LNCs in Transwell) and DF (Cocultured OMECs supported by DF-LNCs in Transwell). Aside from $3 \mathrm{D}$ coculturing, we also cultured OMECs and limbal epithelial progenitor cells (LEPCs) by the suspension method on 5\% Matrigel-coated plastic without the support of LNCs as a control. The medium was changed every 2 days. The expression level of the specific corneal markers CK12 and Pax6 in the cocultured OMECs was also observed. On day 10-14 of coculturing in the Transwell system, we used T/E to digest the OMECs for further RT-PCR and Western blot assay. Furthermore, we placed 5\% Matrigel-coated slides into the insert in advance for immunofluorescence staining to detect expression of CK3, CK12, Pax6, and $\Delta$ Np63 $\alpha$ in $\mathrm{ME}$ and DF.

\section{Renewing LNCs and comparison with 3T3 cells in the Transwell system}

After successful coculturing of OMECs and LNCs in the Transwell system, we observed the ability of LNCs to support the growth and maintain the phenotype of OMECs compared with that of the gold-standard cell line NIH-3T3 (ATCC, Manassas, VA, USA). At the same time, we observed whether renewing the LNCs in the Transwell system could influence the phenotype of the OMECs. $\mathrm{NIH}-3 \mathrm{~T} 3$ cells were also treated with $4 \mu \mathrm{g} / \mathrm{mL}$ mitomycin $\mathrm{C}$ for $2 \mathrm{~h}$ to restrain their growth and served as a feeder layer similarly to LNCs as we described above. LNCs were renewed by passaging at different ratios in advance, with subsequent steps as shown in Fig. 1d. Briefly, the P2 confluent LNCs were passaged at ratios of 1:4, 1:8, and 1:16. When we changed the medium, we moved the insert to the well containing LNCs, which had been passaged at 1:8. In the same way, we moved the insert to the well containing LNCs that had been passaged at 1:16 when we changed the medium the next time. LNCs were renewed at least twice before cocultured OMECs reached confluence. This renewing group was designated DF+. The cornea epithelium was used as a probable positive control. The expression level of the specific corneal markers CK12 and Pax6, proliferation marker Ki67, and stem cell marker $\Delta \mathrm{Np} 63 \alpha$ in the cocultured OMECs was observed. On day 10-14, the cocultured cells were trypsinized by $\mathrm{T} / \mathrm{E}$ for further RT-PCR and Western blotting. All materials used in the isolation and cell culture are included in Additional file 1: File S1.

\section{Airlift culture}

The confluent OMECs cocultured with renewed DF-LNCs in Transwell were cultured for an additional 2 weeks by lowering the compartment containing the medium to the bottom of the insert (Fig. 1e). Cocultured OMECs was embedded in paraffin for hematoxylin and eosin staining and immunofluorescence.

\section{RT-PCR}

RT-PCR was performed following a standard protocol [24]. Briefly, $1 \mathrm{~mL}$ Trizol (Aidlab, Beijing, China) was used to extract total RNA. The RNA was then synthesized by DNase and Hiscript Reverse Transcriptase to synthesize cDNA (RNase H, GeneCopoeia, Rockville, MD, USA). $\beta$-actin primers were used as an internal control. The PCR amplification was performed under the following conditions: denaturation at $50{ }^{\circ} \mathrm{C}$ for $2 \mathrm{~min}$ and $95{ }^{\circ} \mathrm{C}$ for $10 \mathrm{~min}$, followed by 40 cycles at $95{ }^{\circ} \mathrm{C}$ for $30 \mathrm{~s}$ and $60{ }^{\circ} \mathrm{C}$ for $30 \mathrm{~s}$. All RT-PCR experiments were performed in 
triplicate for each group. The primer sequences used in RT-PCR are listed in Additional file 1: File S2.

\section{Western blotting}

Western blotting was performed following a standard method that has been previously reported [25]. Briefly, the protein concentration was determined using the bicinchoninic acid protein assay (Beyotime, Shanghai, China). The same amount of protein in the total cell extract was isolated by $10 \%$ sodium dodecyl sulfate-polyacrylamide gel electrophoresis and transferred to a polyvinylidene fluoride (PVDF) membrane, which was then blocked with 5\% (wt/ vol) fat-free milk. The membrane was continuously incubated with specific primary antibodies and their respective secondary antibodies, with $\beta$-actin or GADPH as a loading control. The immunoreactive bands were detected by chemiluminescent reagents. The results were scanned by BandScan to analyze the grayscale values of the film.

\section{Hematoxylin and eosin staining}

The slides were first stained in Harris' hematoxylin for $8 \mathrm{~min}$. After washing, they were immersed in 1\% acid and then $1 \%$ ammonia for differentiation. The slides were rinsed in water for $1 \mathrm{~h}$ and then rinsed once in distilled water, followed by treatment with $70 \%$ and $90 \%$ alcohol, respectively, for $10 \mathrm{~min}$ for dehydration. They were then stained in eosin-phloxine solution for 2 to $3 \mathrm{~min}$, dried, and observed under a microscope.

\section{Immunohistochemistry}

After being immersed in PBS three times, the slides were treated with $0.5 \%$ Triton $\mathrm{X}-100$ at room temperature, and serum was used to block the antigen. The cells were then incubated with primary and secondary antibodies and subsequently stained with DAPI. Images were acquired with a fluorescence microscope (BX53, Olympus, Tokyo, Japan). All antibodies used in the experiment are included in Additional file 1: File S3.

\section{Statistical analysis}

Data in the figures are shown as the mean \pm SD. At first, one-way analysis of variance (ANOVA) was used to compare the general differences, followed by paired Student's $t$ test if $p<0.05$ to compare two groups for RT-PCR and Western blot experiments. Unpaired Student's $t$ test was used to compare the positive cell rate. $p<0.05$ was considered statistically significant.

\section{Results}

Immunofluorescence of the rat oral mucosa, central cornea, and limbus tissues

The oral mucosa, central cornea, and limbus all expressed CK3 over the entire epithelial layers. However, the oral mucosa did not express CK12 or Pax6, while the central cornea and limbus expressed CK12 and Pax6 over the whole epithelium. Furthermore, the oral mucosa and limbus both expressed $\Delta \mathrm{Np} 63 \alpha$ at the basal layer of the epithelium, whereas the central cornea did not express $\Delta \mathrm{Np} 63 \alpha$ (Fig. 2).

\section{Molecular phenotype characterization of LNCs}

P0 LNCs were double-stained for Vim and CK12, $\triangle \mathrm{Np} 63 \alpha$ or Pax6 in either MESCM or DMEM/F12 supplemented with $10 \%$ fetal bovine serum. The P0 LNCs included both $\mathrm{CK} 12^{+}, \Delta \mathrm{Np} 63 \alpha^{+}$, $\mathrm{Pax}^{+}$and $\mathrm{Vim}^{+}$cells (Fig. 3a). Double immunofluorescence of Vim and CK12, $\Delta \mathrm{Np} 63 \alpha$ or Pax6 in P3 ME-LNCs and DF-LNCs was also assessed to confirm that purified LNCs were obtained from rats. Both P3 ME-LNCs and DF-LNCs were $\mathrm{CK} 12^{-}, \Delta \mathrm{Np} 63 \alpha^{-}, \mathrm{Pax}^{-}, \mathrm{Vim}^{+}, \mathrm{N}$-cadherin ${ }^{+}, \mathrm{Oct}^{+}$, and Sox $2^{+}$, indicating that they had been purified and represented the phenotype of limbal niche cells (Fig. 3b). RT-PCR and Western blot were performed to compare the expression levels of Oct4 and Sox2 between ME-LNCs and DF-LNCs. The expression levels of Oct4 and Sox 2 in ME-LNCs were significantly higher than that in DF-LNCs. The relative mRNA level of Oct4 was $1.363 \pm 0.054$-fold for ME-LNCs compared with DF-LNCs $(p=0.00318)$. The relative mRNA level of Sox2 was $1.904 \pm 0.089$-fold for ME-LNCs compared with DF-LNCs $(p=0.00735)$ (Fig. 3c). Western blotting showed that ME-LNCs expressed higher Oct4 and Sox2 than DF-LNCs at the protein level. Detailed data are provided in Additional file 1: Files S4 and S5.

\section{Transdifferentiation of OMECs into corneal epithelial-like} cells under 3D coculturing conditions

OMECs and LNCs grown on the 3D Matrigel began to form spheres on the first day of coculturing and the spheres became rounder and bigger by day 7 (Fig. 4a), similarly to limbal stem cells and LNCs. We observed that the groups involving SHEM generated a larger sphere than those involving MESCM. Groups involving MESCM generated smaller and rounder spheres. RT-PCR showed that the expression level of CK12 in OMECs, ME-ME, ME-SH, DF-ME, DF-SH, and the cornea was $0.963 \pm 0.092,1.267 \pm 0.058,2.452 \pm 0.262,1.395$ $\pm 0.042,4.572 \pm 0.475$, and $9.216 \pm 0.670$, respectively. No statistically significant difference between OMECs and ME-ME was observed ( $p=0.06837, n=3)$, but OMECs were statistically different from the other groups $(p<0.05$, $n=3)$. When ME-ME was compared with ME-SH, a statistically significant difference was noted ( $p=0.01461, n=3)$, while DF-ME and DF-SH had similar results $(p=0.00871$, $n=3)$. The expression level of CK12 in DF-SH was also upregulated when compared with that of ME-SH $(p=0.03653, n=3)$. In addition, the DF-SH group, which expressed the highest level of CK12, was 


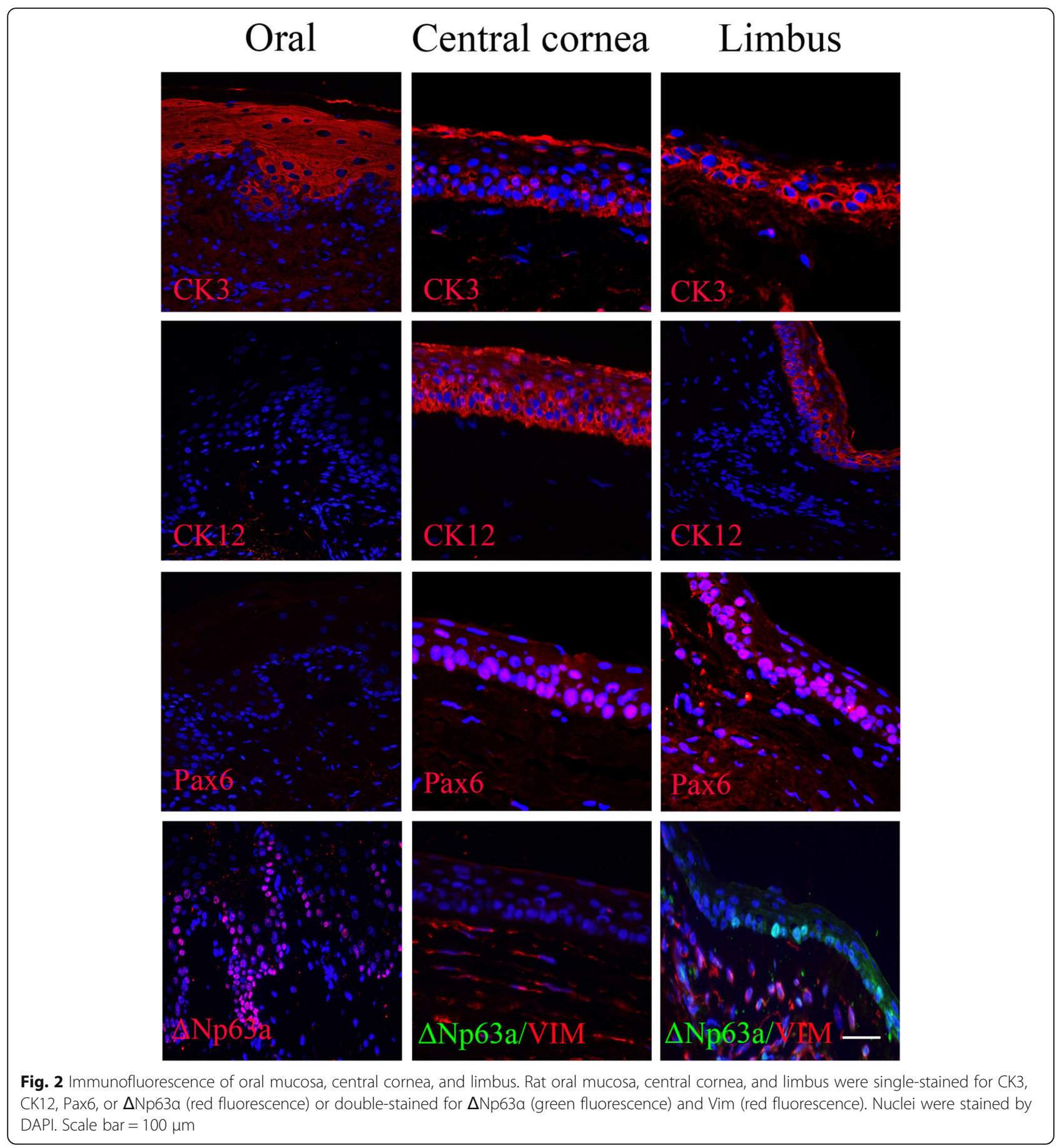

statistically different from the cornea group $(p=0.00073$, $n=3$ ). The expression level of Pax6 in OMECs, ME-ME, ME-SH, DF-ME, DF-SH, and the cornea was $1.071 \pm$ $0.072,1.206 \pm 0.059,3.440 \pm 0.189,1.625 \pm 0.088,7.031 \pm$ 0.782 , and $10.143 \pm 0.830$, respectively. OMECs were statistically different from the other groups $(p<0.05, n=3)$, except for ME-ME $(p=0.21115, n=3)$. Furthermore, when comparing ME-ME with ME-SH and DF-ME with DF-SH, statistical differences were observed $(p=0.00386$ for ME-ME and ME-SH, $p=0.00756$ for DF-ME and DF-SH, $n=3$ ). The expression level of Pax6 in DF-SH was also upregulated when compared with that in ME-SH $(p=0.01067, n=3)$, whereas the expression level in the DF-SH group did not reach that in the cornea $(p=0.01258, n=3)$. Results are shown in Fig. $4 \mathrm{~b}$, and detailed data are included in Additional file 1: File S6. The immunofluorescence results showed that OMECs were largely negative for CK12 and Pax6 
A

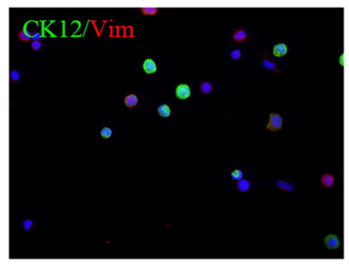

$\mathrm{B}$

ME-LNC
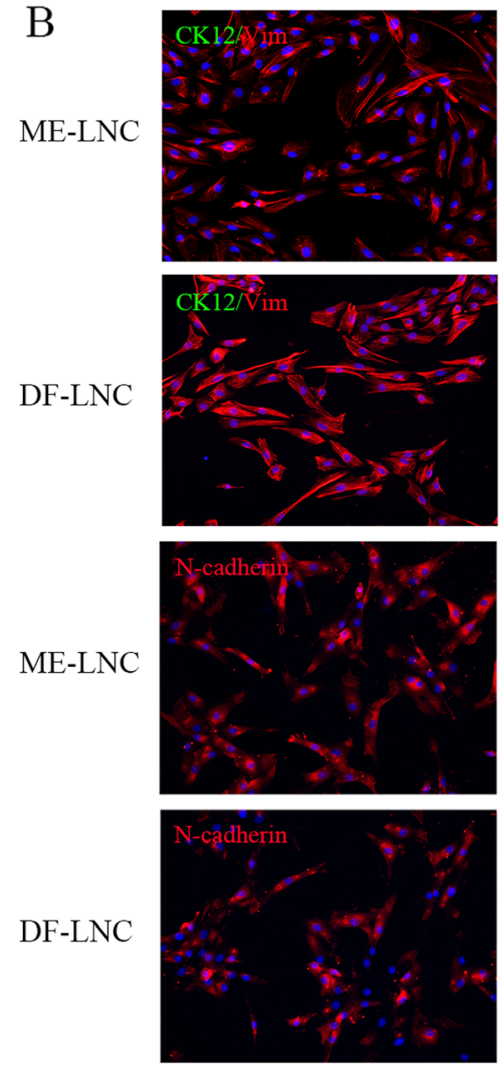

$\mathrm{C}$

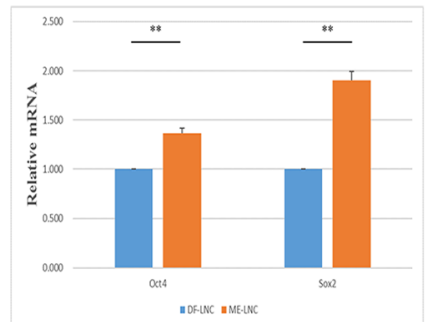

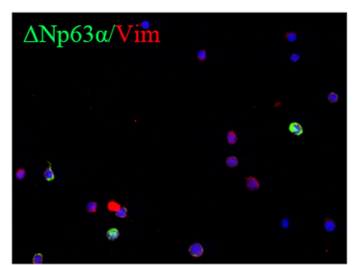
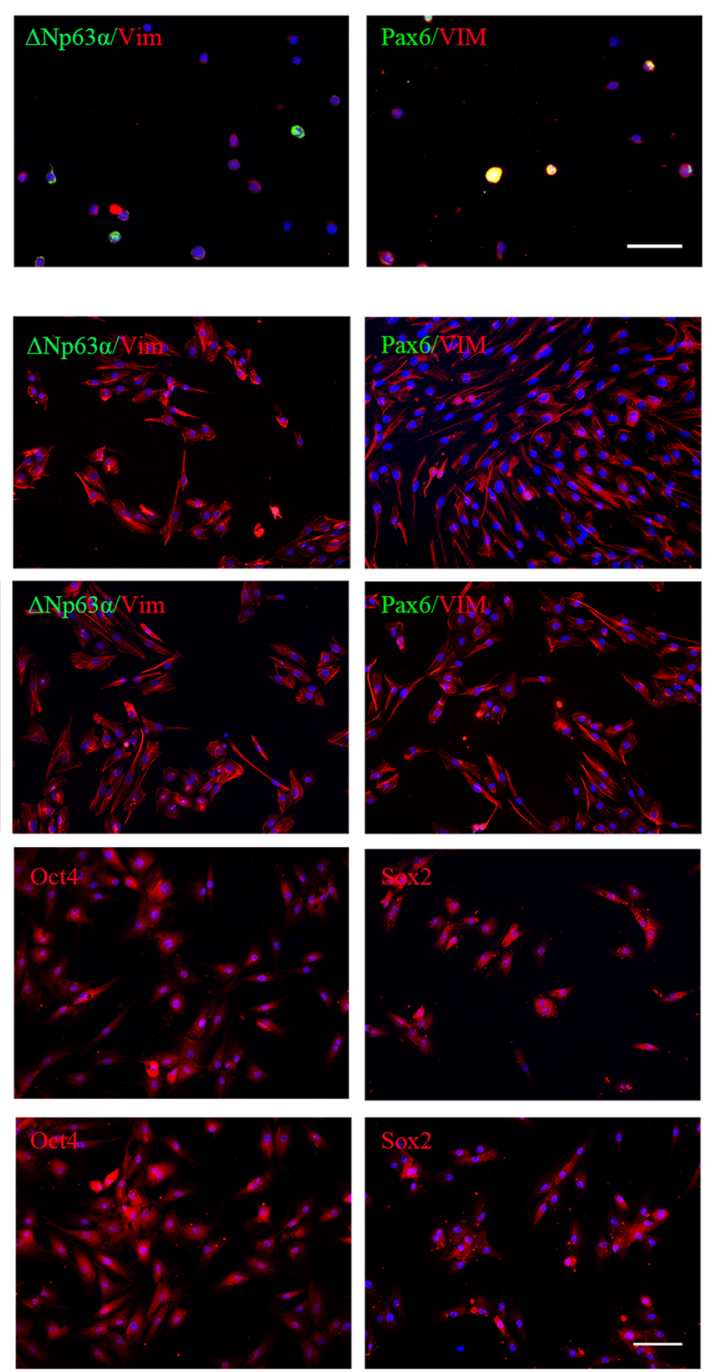

$\mathrm{D}$

DF-LNC ME-LNC

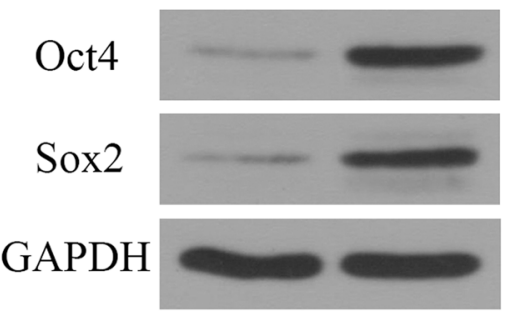

Fig. 3 Molecular phenotype characterization of LNCs. a P0 limbal niche cells (LNCs) were double-stained for Vim (red fluorescence) and CK12 (green fluorescence), $\Delta$ Np63a (green fluorescence) or Pax6 (green fluorescence). b P3 LNCs grown in MESCM (ME-LNCs) and LNCs grown in DMEM/F12 + 10\% FBS (DF-LNCs) were double-stained for Vim (red fluorescence) and CK12 (green fluorescence), $\Delta$ Np63a (green fluorescence) or Pax6 (green fluorescence). Furthermore, they were single-stained for N-cadherin, Oct4, or Sox2 (scale bar $=100 \mu m$ ). c Relative mRNA levels of Oct4 and Sox2 expressed in P3 DF-LNCs and ME-LNCs. $\mathbf{d}$ Western blot analysis of Oct4 and Sox2 expression in P3 DF-LNCs and ME-LNCs; GAPDH was used as a loading control. ${ }^{* *} p<0.01$

expression, while the cornea epithelium was positive for CK12 and Pax6 expression (Fig. 4c). Positive cell counting revealed results very similar to those of RT-PCR, except that ME-ME was statistically different from OMECs regarding the positive rate for both CK12 and Pax6 ( $p=0.04075$ for CK12, $p=0.03035$ for Pax6, $n=3$ ). Furthermore, no statistically significant difference between ME-SH and DF-SH in the positive rate of 


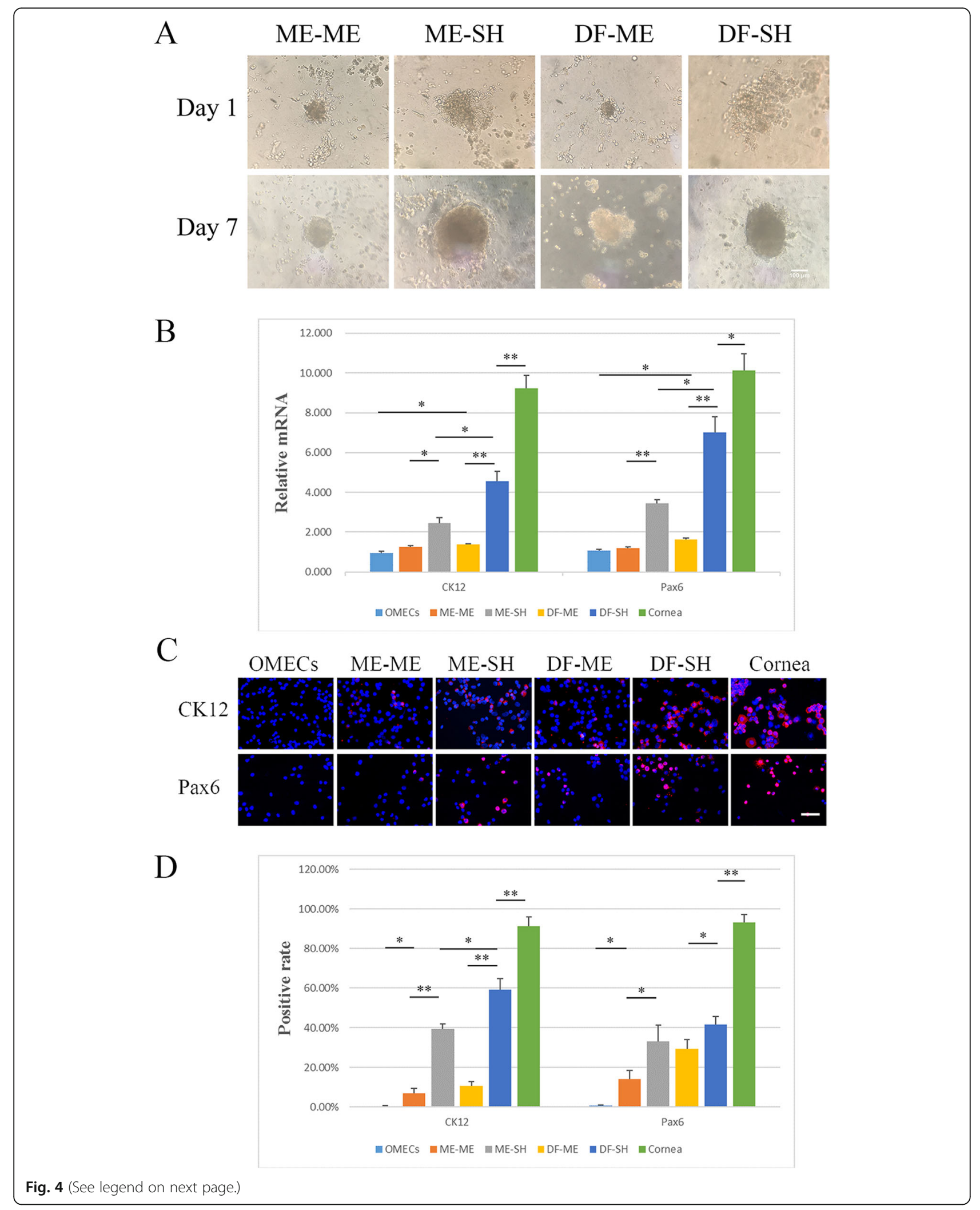


(See figure on previous page.)

Fig. 4 Three-dimensional coculturing of oral mucosal epithelial cells (OMECS) and limbal niche cells (LNCs) under different conditions. a Morphologic characterization of OMECs and LNCs cocultured on 3D Matrigel. b The relative mRNA levels of CK12 and Pax6 in cocultured OMECs and LNCs. c The immunofluorescence of suspended OMECs and LNCs cocultured on 3D Matrigel. Cells were single-stained for CK12 or Pax6 (red fluorescence), and nuclei were stained by DAPI. Scale bar $=100 \mu \mathrm{m}$. $\mathbf{d}$ The positive rate of CK12 and Pax6 expression in cocultured OMECs and LNCs. ${ }^{* *} p<0.01,{ }^{*} p<0.05$. DF-ME LNCs grown in DMEM/F12 (DF-LNCs) 3D cocultured in MESCM, DF-SH DF-LNCs 3D cocultured in SHEM, ME-ME LNCs grown in MESCM (ME-LNCS) 3D cocultured in MESCM, ME-SH ME-LNCs 3D cocultured in SHEM

Pax6 was observed $(p=0.21083, n=3)$. Results for CK12 and Pax6 positivity are shown in Fig. 4d and detailed data can be found in Additional file 1: File S7.

\section{Transwell coculturing of OMECs and LNCs}

According to the results of $3 \mathrm{D}$ coculturing, we chose SHEM as the culture medium for further experiments. However, the LNCs were still obtained by culture in MESCM or DMEM/F12 supplemented with $10 \%$ fetal bovine serum. OMECs cultured in the Transwell system reached confluence at days 10-14. The morphology of OMECs and LEPCs revealed typical slabstone-like features with or without the support of LNCs (Fig. 5a). RT-PCR showed that the transcript levels of CK12 and Pax6 were upregulated for both $\mathrm{ME}$ and DF when compared with that of OMECs (Fig. 5b). The transcript level of CK12 in ME and DF was upregulated by 1.590 \pm 0.139 -fold $(p=0.01650, n=3)$ and $1.817 \pm 0.162$-fold $(p=0.00556, n=3)$ compared with that in OMECs at $1.158 \pm 0.146$. The transcript level of CK12 in DF was even higher than that in $\operatorname{ME}(p=0.00609, n=3)$ and was statistically different compared with that in LEPCs $(3.346 \pm 0.246)$. The transcript level of Pax6 in ME and DF was upregulated by $2.072 \pm 0.451$-fold $(p=0.02621$, $n=3)$ and $2.542 \pm 0.542$-fold $(p=0.01419, n=3)$ compared with that in OMECs at $1.336 \pm 0.294$. The transcript level of Pax6 in DF was even higher than that in $\operatorname{ME}(p=0.04070, n=3)$ and was also statistically different compared with that in LEPCs $(p=0.01312, n=3)$. The protein level of CK12 in DF was higher when compared with that in OMECs and ME ( $p=0.01934$ compared with OMECs, $p=0.01487$ compared with ME, $n=3$ ) but lower compared with that in LEPCs $(p=0.01034, n=3)$. CK12 levels were also higher in ME than in OMECs $(p=0.02748, n=3)$. The protein level of Pax6 in DF was higher when compared with that in OMECs $(p=0.03333$, $n=3)$ and lower than that in LEPCs $(p=0.01626, n=$ 3) but was not statistically different from that in $\mathrm{ME}$ $(p=0.05273, n=3)$. ME also had a higher Pax6 protein level compared with that in OMECs $(p=0.04809$, $n=3)$. The relative protein levels and results of Western blot assay are shown in Fig. $5 \mathrm{c}$ and d, respectively. The immunofluorescence results demonstrated that all groups contained $\mathrm{CK}^{+}$cells, while the cultured OMECs showed almost negative expression of CK12 and Pax6. On the other hand, LEPCs comprised almost all $\mathrm{CK} 12^{+}$and $\mathrm{Pax}^{+}$cells. ME and DF showed pronounced CK12 and Pax6 expression in a portion of cells, according to the morphologic characterization. We also observed that $\mathrm{ME}$ and $\mathrm{DF}$ retained some $\triangle \mathrm{Np} 63 \alpha^{+}$cells, as did OMECs and LEPCs. The immunofluorescence of Transwell cocultured groups is shown in Fig. 5e, and additional data are included in Additional file 1: Files S8 and S9.

\section{Renewing LNCs in the Transwell coculture system and comparison with 3 T3 cells}

After comparing the effect of transdifferentiation in the Transwell system that was supported by ME-LNC or DF-LNC, we chose DF-LNC as the feeder layer for further experiments, while 3T3 cells were used as a control. LNCs cocultured with OMECs in all groups showed a typical slabstone-like morphology (Fig. 6a). The transcript level of CK12 was upregulated by $2.080 \pm 0.183$-fold and $1.542 \pm$ 0.086 -fold in the DF+ and DF groups, respectively, when compared with that in the 3T3 group $(p<0.05, n=3)$. DF + was also statistically different when compared with DF and the cornea (all $p<0.05, n=3$ ). The transcript level of Pax6 was upregulated by $2.255 \pm 0.289$-fold and $1.681 \pm$ 0.166 -fold in the DF+ and DF groups, respectively, when compared with that in the 3T3 group $(p<0.05, n=3)$. Furthermore, the expression level of Pax6 in DF+ was statistically different from that in DF and the cornea (all $p<0.05$, $n=3)$. The relative mRNA level of Ki67 was upregulated by $2.010 \pm 0.262$-fold and $1.488 \pm 0.088$-fold in the $\mathrm{DF}+$ and DF groups, respectively, compared with that in the 3T3 group $(p<0.05, n=3)$. Specifically, Ki67 expression was most pronounced in the DF+ group. Moreover, the expression level of $\Delta \mathrm{Np} 63 \alpha$ was highest in the 3T3 group when compared with that in the DF+ and DF groups $(p<0.01, n=3)$, while DF+ showed the lowest expression of $\Delta \mathrm{Np} 63 \alpha$ (Fig. 6b). Western blot showed similar results regarding protein expression of CK12, Pax6, and Ki67, except there were no statistically significant differences between $3 \mathrm{~T} 3$ and DF at the protein level of Pax6 ( $p=0.06595, n=3)$ (Fig. 6c, d). In conflict with the results of RT-PCR, DF+ was higher than the DF group at the protein level for $\Delta \operatorname{Np} 63 \alpha(p=$ $0.02418, n=3)$, though they were both statistically significant different from 3T3 $(p<0.01, n=3)$. Further data and analysis are included in Additional file 1: Files S10 and S11. 


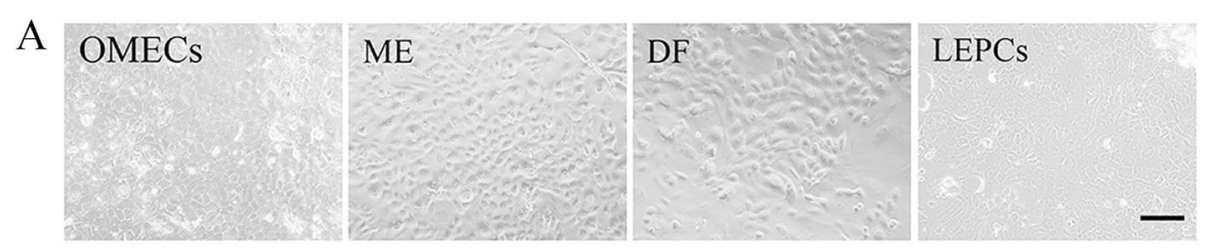

B

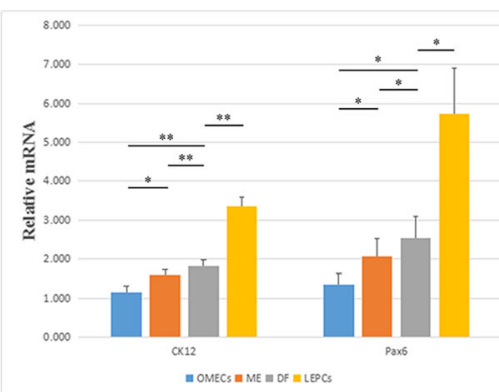

C

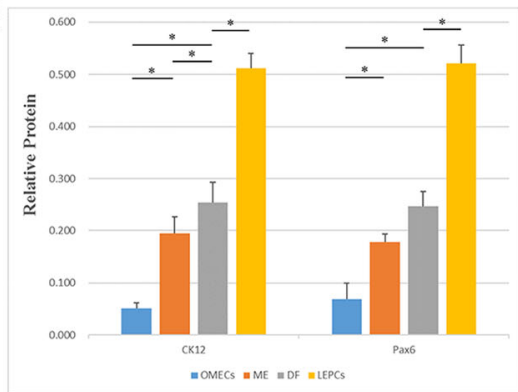

D

\section{OMECs ME DF LEPCs}

CK12

Pax6

$\beta$-actin

E
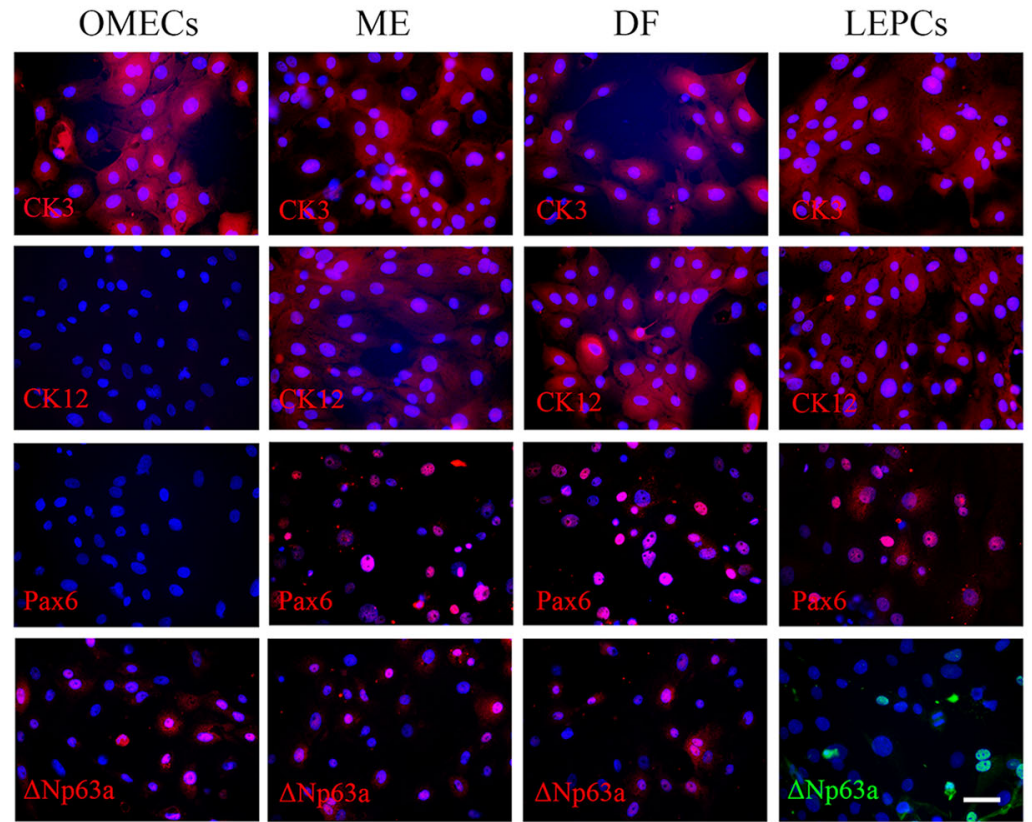

Fig. 5 Transwell coculturing of oral mucosal epithelial cells (OMECs) and limbal niche cells (LNCS) in supplemented hormonal epithelial medium (SHEM). a Morphologic characterization of OMECs and LNCs cocultured in Transwell. Scale bar $=100 \mu \mathrm{m}$. $\mathbf{b}$ The relative mRNA expression of CK12 and Pax6 in cocultured OMECs and LNCs. c The relative protein levels of cocultured OMECs and LNCs. $\mathbf{d}$ Western blot analysis of CK12 and Pax6 expression in cocultured OMECs and LNCs; $\beta$-actin was used as an internal control. e Cells were single-stained for CK3, CK12, Pax6, and $\Delta N p 63 a$, and nuclei were stained by DAPI. Scale bar $=50 \mu \mathrm{m} .{ }^{* *} p<0.01,{ }^{*} p<0.05$. DF Cocultured OMECs supported by DF-LNCs in Transwell, LEPC Limbal epithelial progenitor cell, ME Cocultured OMECs supported by ME-LNCs in Transwell 


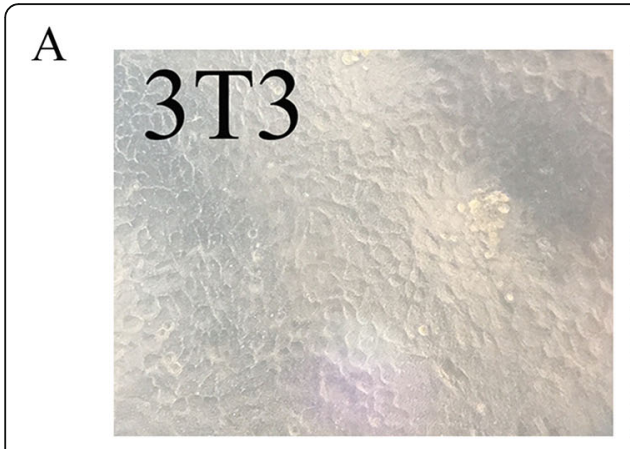

B

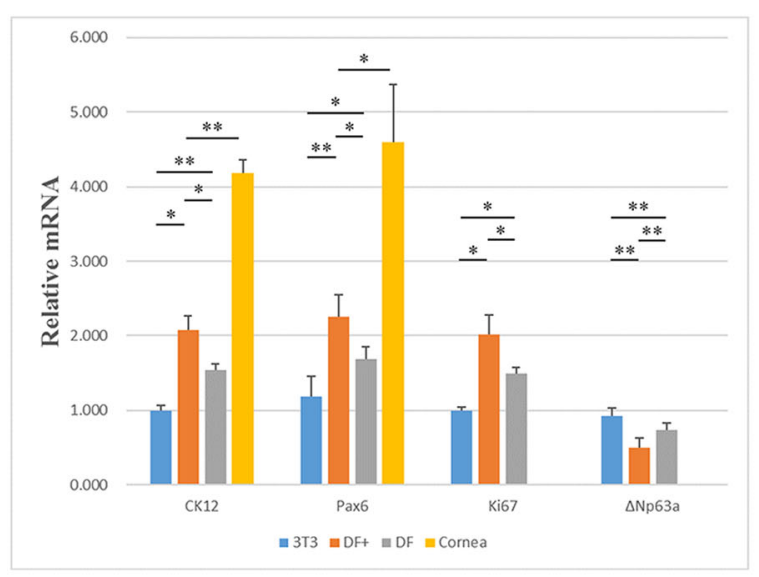

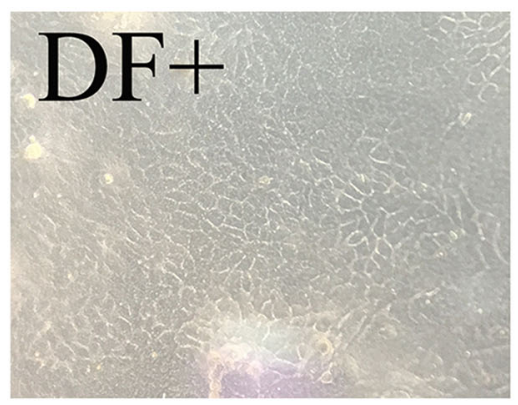

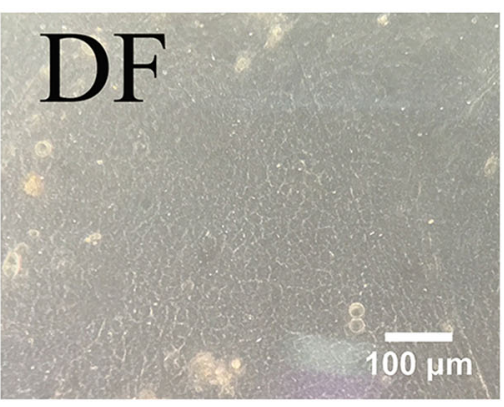

C

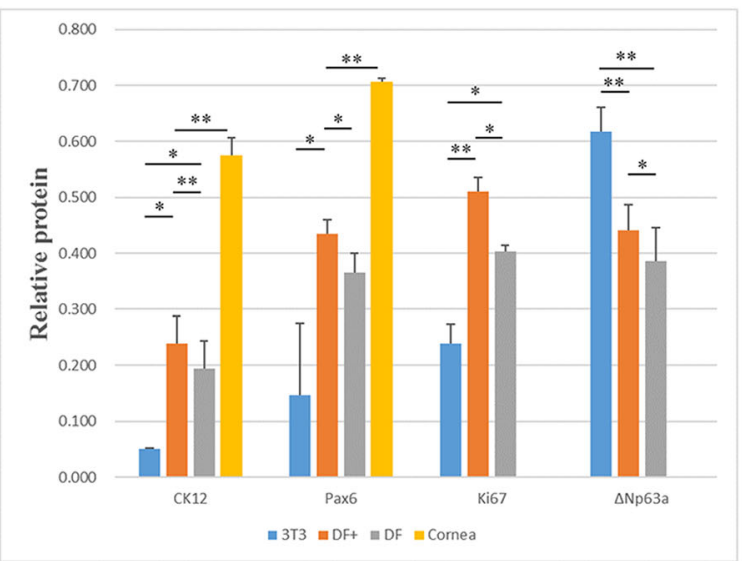

D

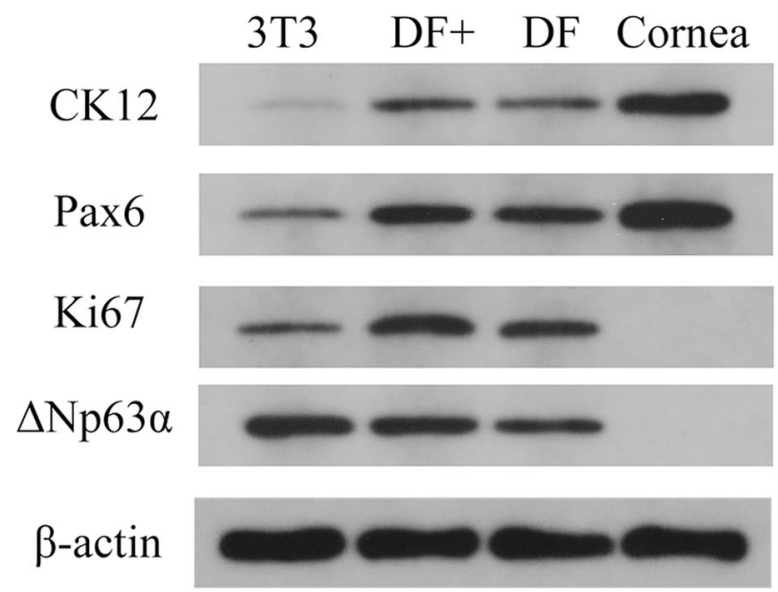

Fig. 6 Renewing the DF-LNCs in the Transwell system and comparison with $3 T 3$ cells. a Morphologic characterization of OMECs supported by $3 T 3$ cells (3T3), DF-LNC (DF), and renewed DF-LNC (DF+) in Transwell. Scale bar = $100 \mu \mathrm{m}$. b The relative mRNA expression of CK12, Pax6, Ki67, and $\Delta$ Np63a expressed by OMECs supported by 3 T3 cells, DF-LNC, and renewed DF-LNC in Transwell. c The relative protein level of CK12, Pax6, Ki67, and $\Delta$ Np63a expressed by OMECs supported by 3T3 cells, DF-LNC, and renewed DF-LNC in Transwell. d Western blot analysis of CK12, Pax6, Ki67, and $\Delta$ Np63a; $\beta$ actin was used as an internal control. ${ }^{* *} p<0.01,{ }^{*} p<0.05$

\section{Airlift culture}

The hematoxylin and eosin staining of airlift cocultured OMECs showed stratified structures for 2-3 layers. The entire layer of the stratified epithelium expressed CK3 and CK12; however, this was negative for Pax6 and $\Delta \mathrm{Np} 63 \alpha$ (Fig. 7).
A flowchart describing the steps involved in confirmation of transdifferentiation of OMECs is provided in Fig. 8.

\section{Discussion}

In this study, we achieved the transdifferentiation of rat OMECs into corneal epithelial-like cells with the help of 

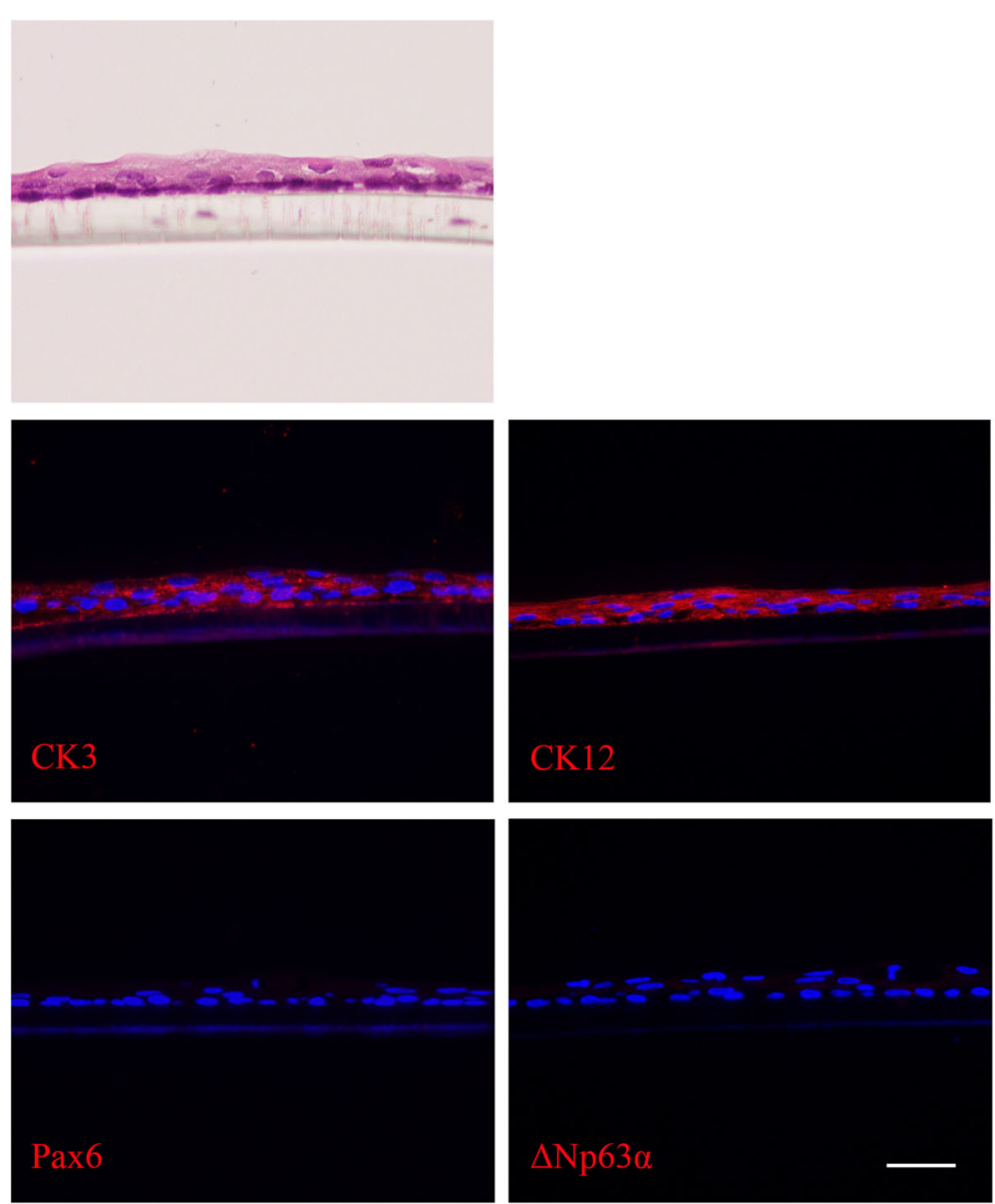

Fig. 7 Hematoxylin and eosin staining and immunofluorescence of airlift cocultured OMECs. Cocultured OMECs were stained with hematoxylin and eosin and single-stained for CK3, CK12, Pax6, and $\Delta$ Np63a. Scale bar $=100 \mu \mathrm{m}$

LNCs in vitro. Moreover, we demonstrated that LNCs as well as 3T3 cells have the ability to support OMECs.

At the beginning of our study, we evaluated the immunofluorescence of the oral mucosa, central cornea, and limbus to prove that only CK12 and Pax6 could be defined as cornea-specific markers, as previously reported [26-28]. We agree with previous studies [23] suggesting that CK3 cannot be considered a cornea-specific marker, and CK3/12 is a marker of epithelium differentiation. Furthermore, we also confirmed that oral mucosa and limbus express the same stem cell marker, $\Delta \mathrm{Np} 63 \alpha$, as previously described [29].

LNCs have been involved in many studies; these cells are localized in the limbus subjacent to the epithelial basement membrane and maintain a close association with limbal epithelial stem cells $[20,21,25,30]$. Several methods to isolate LNCs have been described [20, 25, 31], but the current consensus is that digestion of limbal tissue with collagenase results in optimal LNCs [32]. Vimentin was the first marker identified at the base of the limbus [33]. Oct4 plays an important role in maintaining the pluripotency and self-renewal of embryonic stem cells, and it has been proven to be expressed in the basal limbus [34]. Sox 2 is also considered a marker of LNCs [35], and $\mathrm{N}$-cadherin has been detected in LNCs and melanocytes [36]. Therefore, we performed double immunofluorescence staining on P3 LNCs to confirm that we had obtained purified cells, which represented the phenotype of limbal niche cells, cultured in either MESCM or DMEM/ F12 supplemented with $10 \%$ fetal bovine serum. As a result, LNCs did not interfere with the results of further coculture. The results of RT-PCR and Western blotting regarding Oct4 and Sox 2 expression in LNCs indicated that using MESCM for culturing rather than DMEM/F12 supplemented with $10 \%$ fetal bovine serum could produce LNCs that expressed more mesenchymal stem cell markers, as previously reported [20].

Three-dimensional cocultured OMECs and LNCs produced spheres owing to the 3D Matrigel [37], and other studies have confirmed that LNCs have the ability to attract and aggregate the epithelium [20, 21]. Results of 3D coculturing demonstrated that use of SHEM and 

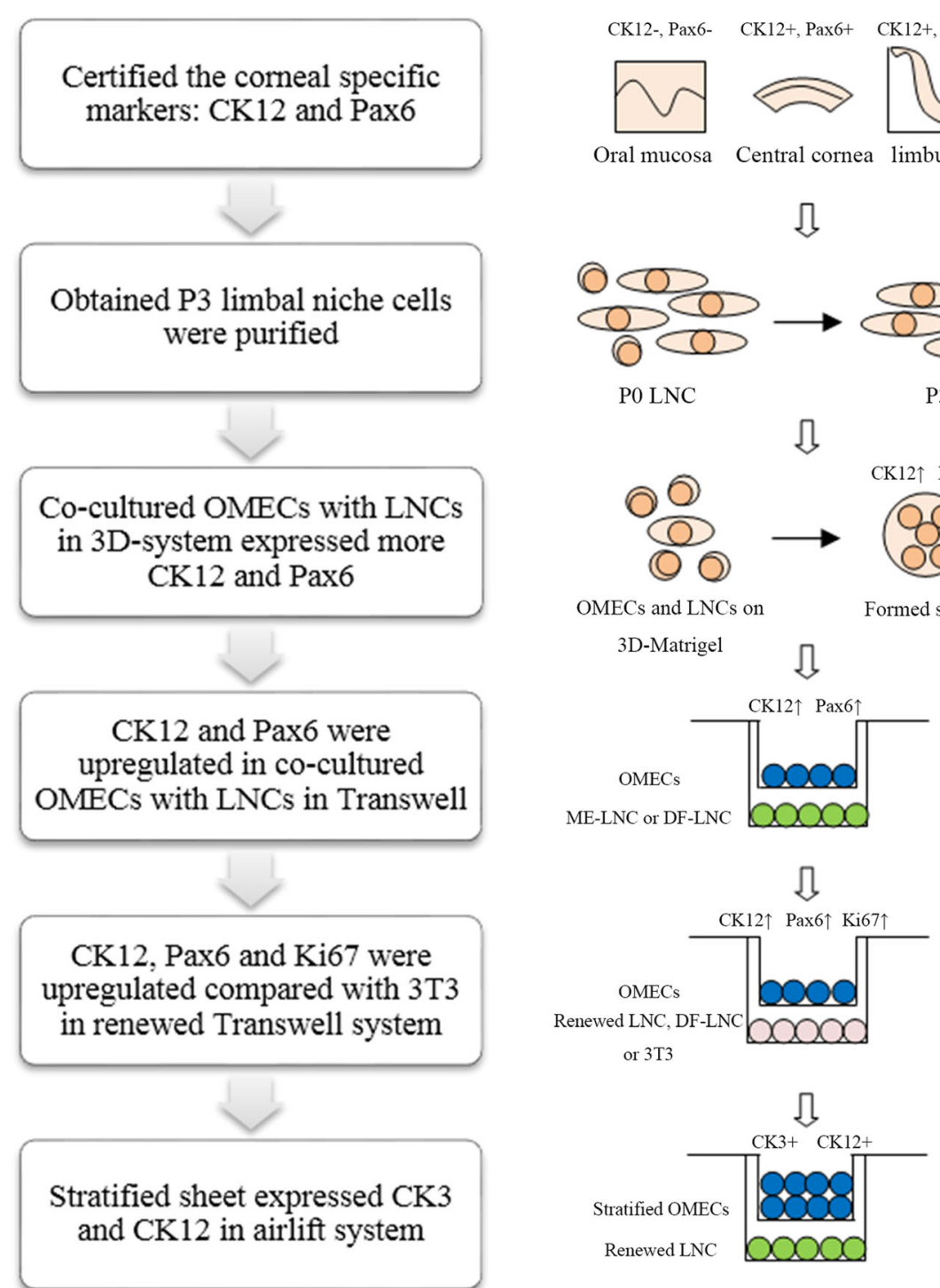

Fig. 8 Flow chart describing the steps involved in confirmation of transdifferentiation of OMECs. This flowchart shows the logic flow behind arriving at the results that we have presented and the conclusions drawn from those results. DF-LNC LNCs grown in DMEM/F12 + 10\% FBS, LNC limbal niche cell, ME-LNC LNCs grown in MESCM, OMEC oral mucosal epithelial cell

DF-LNCs could upregulate the expression of CK12 and Pax6, indicating that they are better for transdifferentiation of OMECs to corneal epithelial-like cells. However, MESCM is not suitable for transdifferentiation. We consider these results to be due to the ability of MESCM to maintain the phenotype of stem cells and prevent their differentiation $[20,21,25,38]$. Furthermore, we also demonstrated that maintaining the phenotype of LNCs does not benefit transdifferentiation. We consequently attempted to coculture OMECs and LNCs in the Transwell system to obtain a transplantable epithelium sheet. MESCM failed to support the growth of OMECs in the early period of the study, forcing us to abandon this medium in the Transwell system.
When we compared the transdifferentiation effect of ME-LNCs and DF-LNCs in the Transwell system, we observed results similar to those obtained with the 3D coculturing system, showing that DF-LNCs were more effective than ME-LNCs. Immunofluorescence assay of cultured OMECs, ME, DF, and LEPCs confirmed that CK3 cannot be defined as a cornea-specific marker, whereas higher expression levels of CK12 and Pax6 confirmed the transdifferentiation of OMECs into corneal epithelial-like cells after coculturing with ME-LNCs or DF-LNCs. Moreover, we noticed that some $\Delta \mathrm{Np} 63 \alpha^{+}$cells remained after coculturing, prompting us to compare the OMEC support by LNCs and 3T3 cells, which are considered the gold standard for the feeder layer. At the same time, we attempted 
to renew the LNCs in the culture system. 3T3 cells are considered the gold standard for the feeder layer because of their ability to support the proliferation and maintain the phenotype of OMECs $[9,10,12]$. The expression level of CK12, Pax6, and Ki67 was also upregulated when compared with that in other groups by renewing the LNCs in the Transwell coculturing system, suggesting that this might be a potential method for improving the efficiency of transdifferentiation and proliferation of OMECs. However, $\triangle \mathrm{Np} 63 \alpha$ expression was different between the DF+ and DF groups at both the transcript and protein level, although it was most pronounced in the 3T3 group. We considered this outcome the effect of complex molecular mechanisms in transdifferentiation, which requires further study. The obtained stratified cocultured OMECs showed 2-3 layers and expressed CK3 and CK12, indicating that they were well differentiated, and had a corneal epithelial-like phenotype, which might be a potential source for ocular reconstruction. In conflict with a previous study [16], Pax6 and $\Delta \mathrm{Np} 63 \alpha$ were negative, probably because of long-term culturing by the airlifting method [28, 37], although they were both pronounced when cocultured OMECs reached confluence.

We also noticed that 3T3 cocultured OMECs did not show as much negative expression, especially for Pax6, as cultured OMECs with no feeder layer. Other researchers have encountered a similar situation [23]; however, no evidence has indicated that 3T3 cells cocultured with OMECs can upregulate the expression of Pax6. Even so, coculturing with LNCs on a 3D Matrigel or in the Transwell system upregulated the expression of CK12 and Pax6 when compared with that in OMECs alone or cocultured with 3T3, which conflicts with a previous study [23]. We believe that the culturing system and method of LNC acquisition are key determiners. In the previous study, the researchers used a direct-contact coculture system in which OMECs were merged with limbal fibroblast cells, and did not obtain positive results. In this direct-contact system, feeder cells were very difficult to exchange, although the researchers described a T/E digestion method in which the OMECs would be trypsinized every time they changed the feeder layers. To our understanding, other literature focusing on adult stem cell transdifferentiation into corneal epithelial-like cells is based on a limbal niche environment offered by conditioned medium, which consisted of the medium that had been used for limbal niche cell culture $[15,18,19]$. We improved this method using the Transwell system, which was loaded with fresh conditioned medium and not frozen. This 0.4- $\mu \mathrm{m}$ Transwell system can reliably prevent cells in the lower chamber from entering the upper chamber [39]. The 3D Matrigel coculture system is a very advanced coculturing system used to investigate intercellular signaling and pathways that can simulate the niche microenvironment in vitro [21, 30]. Although we merged OMECs and LNCs in this system, we verified that we obtained purified LNCs that would not produce interference, and LNCs in this system did not require changing. Furthermore, the obtained niche cells were different from limbal fibroblast cells as previously described [31]. Other researchers have used a special matrix [40] or factors [41] to achieve transdifferentiation of OMECs into corneal-like epithelium, support for which we believe requires further evidence. Although we succeeded in transdifferentiating rat OMECs into corneal epithelial-like cells in vitro, whether the same can occur in human cells is unknown and will be investigated by us in the future.

Very recently, keratoplasty lenticules, a novel carrier, have been proven to be suitable for transplantation to reconstruct the ocular surface and treat LSCD [42]. Our research team has succeeded in culturing OMECs on acellular porcine corneal stroma (APCS) [24], and a clinical trial in our hospital has demonstrated that APCS transplantation is useful for treating fungal keratitis [43]. Based on these investigations, we are also attempting to coculture OMECs on APCS with LNCs as feeder layers in a Transwell system for further transplantation in animal experiments (Additional file 1: File S12). This treatment method appears promising for the treatment of LSCD in the future.

\section{Conclusion}

In summary, we achieved transdifferentiation of OMECs into corneal epithelial-like cells in vitro. We evaluated two coculturing systems: 3D Matrigel for investigation of signaling pathways between OMECs and LNCs and a Transwell system for culturing a transplantable epithelium sheet. These cells have the potential to serve as an alternative source for transplantation. This investigation is of great significance for the treatment of LSCD and ocular surface reconstruction.

\section{Additional file}

Additional file 1: S1. The materials used in cell isolation and culture. S2. The primer sequences used in RT-PCR. S3. The antibodies used in the experiments. S4. PCR of DF-LNC and ME-LNC. S5. Western blot of DF-LNC and ME-LNC. S6. PCR of 3D cocultured OMECs and LNCs. S7. Cell counting of 3D cocultured OMECs and LNCs. S8. PCR of Transwell cultured groups. S9. Western blot of Transwell cultured groups. S10. PCR of 3T3 cells and renewed LNCs cultured in Transwell. S11. Western blot of 3T3 cells and renewed LNCs cultured in Transwell. S12. Cocultured OMECs on APCS. S13. IACUC. (ZIP $6676 \mathrm{~kb})$

\section{Abbreviations}

APCS: Acellular porcine corneal stroma; DF+: Cocultured OMECs supported by renewed DF-LNCs in Transwell; DF: Cocultured OMECs supported by DFLNCs in Transwell; DF-LNCs: LNCs grown in DMEM/F12 supplemented with 10\% fetal bovine serum; DF-ME: DF-LNCs 3D cocultured in MESCM; DFSH: DF-LNCS 3D cocultured in SHEM; DMEM/F12: Dulbecco's modified Eagle's medium; LEPC: Limbal epithelial progenitor cell; LNC: Limbal niche cell; 
LSCD: Limbal stem cell deficiency; ME: Cocultured OMECs supported by MELNCs in Transwell; ME-LNCs: LNCs grown in MESCM; ME-ME: ME-LNCs 3D cocultured in MESCM; MESCM: Modified embryonic stem cell medium; ME-SH: ME-LNCS 3D cocultured in SHEM; OMEC: Oral mucosal epithelial cell; PBS: Phosphate-buffered saline; RT-PCR: Reverse-transcription polymerase chain reaction; SHEM: Supplemented hormonal epithelial medium; T/E: 0.25\% trypsin/0.02\% ethylenediaminetetraacetic acid

\section{Acknowledgements}

We would like to thank Editage for English language editing.

\section{Funding}

This study was supported by the National Natural Science Foundation of China (grant nos. 81300736 and 81370993). Adequate funds were provided by this foundation for the purchase of reagents and consumables.

\section{Availability of data and materials}

All data generated and/or analyzed during this study are included in this published article (and Additional file 1).

\section{Authors' contributions}

$X-Y Z$ contributed to the collection and assembly of data, data analysis and interpretation, and manuscript writing. H-TX was responsible for the conception and design of the study and manuscript writing. C-YD and $J L$ contributed to the revision of the manuscript. M-CZ was responsible for the conception, design and interpretation, and final approval of the manuscript. All authors read and approved the final manuscript.

\section{Ethics approval and consent to participate}

This study was conducted after obtaining approval from the Institutional Animal Care and Use Committee at Tongji Medical College, Huazhong University of Science and Technology (IACUC number S760, 9 September 2016; Additional file 1: File S13).

\section{Consent for publication}

Not applicable.

\section{Competing interests}

The authors declare that they have no competing interests.

\section{Publisher's Note}

Springer Nature remains neutral with regard to jurisdictional claims in published maps and institutional affiliations.

\section{Author details \\ 'Department of Ophthalmology, Union Hospital, Tongji Medical College, Huazhong University of Science and Technology, Wuhan 430022, China. ${ }^{2}$ Department of Ophthalmology, Tongji Hospital, Tongji Medical College, Huazhong University of Science and Technology, Wuhan 430022, China.}

Received: 5 July 2018 Revised: 24 August 2018

Accepted: 27 August 2018 Published online: 26 September 2018

\section{References}

1. Lavker RM, Tseng SCG, Sun T-T. Corneal epithelial stem cells at the limbus: looking at some old problems from a new angle. Exp Eye Res. 2004;78:433-46.

2. Pellegrini $G$, Traverso CE, Franzi AT, Zingirian M, Cancedda R, De Luca M Long-term restoration of damaged corneal surfaces with autologous cultivated corneal epithelium. Lancet. 1997;349:990-3.

3. Tsai RJ, Li LM, Chen JK. Reconstruction of damaged corneas by transplantation of autologous limbal epithelial cells. N Engl J Med. 2000;343:86-93.

4. Rama P, Matuska S, Paganoni G, Spinelli A, De Luca M, Pellegrini G. Limbal stem-cell therapy and long-term corneal regeneration. N Engl J Med. 2010; 363:147-55.

5. Jenkins C, Tuft S, Liu C, Buckley R. Limbal transplantation in the management of chronic contact-lens-associated epitheliopathy. Eye. 1993;7: 629-33.

6. Li DQ, Wang Z, Yoon KC, Bian F. Characterization, isolation, expansion and clinical therapy of human corneal epithelial stem/progenitor cells. J Stem Cells. 2014;9:79-91.
7. Shang $X$, Zhang M. Body and organ donation in Wuhan, China. Lancet. 2010;376:1033-4

8. Ilari L, Daya SM. Long-term outcomes of keratolimbal allograft for the treatment of severe ocular surface disorders. Ophthalmology. 2002;109:1278-84.

9. Nishida K, Yamato M, Hayashida Y, Watanabe K, Yamamoto K, Adachi E, Nagai S, Kikuchi A, Maeda N, Watanabe H, et al. Corneal reconstruction with tissue-engineered cell sheets composed of autologous oral mucosal epithelium. N Engl J Med. 2004;351:1187-96.

10. Kolli S, Ahmad S, Mudhar HS, Meeny A, Lako M, Figueiredo FC. Successful application of ex vivo expanded human autologous oral mucosal epithelium for the treatment of total bilateral limbal stem cell deficiency. Stem Cells. 2014;32:2135-46.

11. Utheim TP, Utheim OA, Khan QE, Sehic A. Culture of oral mucosal epithelial cells for the purpose of treating limbal stem cell deficiency. J Funct Biomater. 2016:7(1):5. http://www.mdpi.com/2079-4983/7/1/5.

12. Utheim TP. Concise review: transplantation of cultured oral mucosal epithelial cells for treating limbal stem cell deficiency-current status and future perspectives. Stem Cells. 2015;33:1685-95

13. Inatomi T, Nakamura T, Koizumi N, Sotozono C, Yokoi N, Kinoshita S. Midterm results on ocular surface reconstruction using cultivated autologous oral mucosal epithelial transplantation. Am J Ophthalmol. 2006; 141:267-275.e261

14. Sharma SM, Fuchsluger T, Ahmad S, Katikireddy KR, Armant M, Dana R, Jurkunas UV. Comparative analysis of human-derived feeder layers with 3T3 fibroblasts for the ex vivo expansion of human limbal and oral epithelium. Stem Cell Rev. 2012:8:696-705.

15. Ahmad S, Stewart R, Yung S, Kolli S, Armstrong L, Stojkovic M, Figueiredo F, Lako M. Differentiation of human embryonic stem cells into corneal epithelial-like cells by in vitro replication of the corneal epithelial stem cell niche. Stem Cells. 2007:25:1145-55.

16. Blazejewska EA, Schlotzer-Schrehardt U, Zenkel M, Bachmann B, Chankiewitz E, Jacobi C, Kruse FE. Corneal limbal microenvironment can induce transdifferentiation of hair follicle stem cells into corneal epithelial-like cells. Stem Cells. 2009:27:642-52

17. Meyer-Blazejewska EA, Call MK, Yamanaka O, Liu H, Schlotzer-Schrehardt U, Kruse FE, Kao WW. From hair to cornea: toward the therapeutic use of hair follicle-derived stem cells in the treatment of limbal stem cell deficiency. Stem Cells. 2011:29:57-66.

18. Gopakumar V, Chatterjee N, Parameswaran S, Nirmala S, Krishnakumar S. In vitro transdifferentiation of human skin keratinocytes to corneal epithelial cells. Cytotherapy. 2016;18:673-85.

19. Cieslar-Pobuda A, Rafat M, Knoflach V, Skonieczna M, Hudecki A, Malecki A, Urasinska E, Ghavami S, Los MJ. Human induced pluripotent stem cell differentiation and direct transdifferentiation into corneal epithelial-like cells. Oncotarget. 2016;7:42314-29.

20. Xie HT, Chen SY, Li GG, Tseng SC. Isolation and expansion of human limbal stromal niche cells. Invest Ophthalmol Vis Sci. 2012:53:279-86.

21. Li J, Chen SY, Zhao XY, Zhang MC, Xie HT. Rat limbal niche cells prevent epithelial stem/progenitor cells from differentiation and proliferation by inhibiting notch signaling pathway in vitro. Invest Ophthalmol Vis Sci. 2017; 58:2968-76.

22. Gonzalez S, Deng SX. Presence of native limbal stromal cells increases the expansion efficiency of limbal stem/progenitor cells in culture. Exp Eye Res. 2013;116:169-76.

23. O'Callaghan AR, Morgan L, Daniels JT, Lewis MP. Human-derived feeder fibroblasts for the culture of epithelial cells for clinical use. Regen Med. 2016;11:529-43.

24. Wang JS, Xie HT, Zhang MC. Characterization of ex vivo expanded oral mucosal epithelium cells on acellular porcine corneal stroma for ocular surface reconstruction. J Ophthalmol. 2017;2017:6761714.

25. Li GG, Zhu YT, Xie HT, Chen SY, Tseng SC. Mesenchymal stem cells derived from human limbal niche cells. Invest Ophthalmol Vis Sci. 2012;53:5686-97.

26. Liu CY, Zhu G, Converse R, Kao CW, Nakamura H, Tseng SC, Mui MM, Seyer J, Justice MJ, Stech ME, et al. Characterization and chromosomal localization of the cornea-specific murine keratin gene Krt1.12. J Biol Chem. 1994;269:24627-36.

27. Chen WYW, Mui MM, Kao WWY, Liu C-Y, Tseng SCG. Conjunctival epithelial cells do not transdifferentiate in organotypic cultures: expression of K12 keratin is restricted to corneal epithelium. Curr Eye Res. 2009;13:765-78.

28. Funderburgh ML, Du Y, Mann MM, SundarRaj N, Funderburgh JL. PAX6 expression identifies progenitor cells for corneal keratocytes. FASEB J. 2005; 19:1371-3. 
29. Pellegrini G, Dellambra E, Golisano O, Martinelli E, Fantozzi I, Bondanza S, Ponzin D, McKeon F, De Luca M. p63 identifies keratinocyte stem cells. Proc Natl Acad Sci U S A. 2001;98:3156-61.

30. Han B, Chen SY, Zhu YT, Tseng SC. Integration of BMP/Wnt signaling to control clonal growth of limbal epithelial progenitor cells by niche cells. Stem Cell Res. 2014;12:562-73.

31. Li Y, Inoue T, Takamatsu F, Kobayashi T, Shiraishi A, Maeda N, Ohashi Y, Nishida K. Differences between niche cells and limbal stromal cells in maintenance of corneal limbal stem cells. Invest Ophthalmol Vis Sci. 2014; 55:1453-62.

32. Funderburgh JL, Funderburgh ML, Du Y. Stem cells in the limbal stroma. Ocul Surf. 2016;14:113-20.

33. Shanmuganathan VA, Foster T, Kulkarni BB, Hopkinson A, Gray T, Powe DG, Lowe J, Dua HS. Morphological characteristics of the limbal epithelial crypt. Br J Ophthalmol. 2007:91:514-9.

34. S-y Z, Zhang C, Baradaran E, Chuck RS. Human corneal basal epithelial cells express an embryonic stem cell marker OCT4. Curr Eye Res. 2010;35:978-85.

35. Chen SY, Hayashida Y, Chen MY, Xie HT, Tseng SC. A new isolation method of human limbal progenitor cells by maintaining close association with their niche cells. Tissue Eng Part C Methods. 2011;17:537-48.

36. Hayashi $R$, Yamato M, Sugiyama H, Sumide T, Yang J, Okano T, Tano $Y$, Nishida K. N-cadherin is expressed by putative stem/progenitor cells and melanocytes in the human limbal epithelial stem cell niche. Stem Cells. 2007;25:289-96.

37. Du Y, Funderburgh ML, Mann MM, SundarRaj N, Funderburgh JL. Multipotent stem cells in human corneal stroma. Stem Cells. 2005:23:1266-75.

38. Nakatsu MN, Gonzalez S, Mei H, Deng SX. Human limbal mesenchymal cells support the growth of human corneal epithelial stem/progenitor cells. Invest Ophthalmol Vis Sci. 2014;55:6953-9.

39. Kim S, Ahn SE, Lee JH, Lim DS, Kim KS, Chung HM, Lee SH. A novel culture technique for human embryonic stem cells using porous membranes. Stem Cells. 2007;25:2601-9.

40. Irani YD, Klebe S, McInnes SJP, Jasieniak M, Voelcker NH, Williams KA. Oral mucosal epithelial cells grown on porous silicon membrane for transfer to the rat eye. Sci Rep. 2017;7:10042

41. Dhamodaran K, Subramani M, Matalia H, Jayadev C, Shetty R, Das D. One for all: a standardized protocol for ex vivo culture of limbal, conjunctival and oral mucosal epithelial cells into corneal lineage. Cytotherapy. 2016;18:546-61.

42. Barbaro V, Nasti AA, Raffa P, Migliorati A, Nespeca P, Ferrari S, Palumbo E, Bertolin M, Breda C, Miceli F, et al. Personalized stem cell therapy to correct corneal defects due to a unique homozygous-heterozygous mosaicism of ectrodactyly-ectodermal dysplasia-clefting syndrome. Stem Cells Transl Med. 2016;5:1098-105.

43. Zhang MC, Liu X, Jin Y, Jiang DL, Wei XS, Xie HT. Lamellar keratoplasty treatment of fungal corneal ulcers with acellular porcine corneal stroma. Am J Transplant. 2015;15:1068-75.

Ready to submit your research? Choose BMC and benefit from:

- fast, convenient online submission

- thorough peer review by experienced researchers in your field

- rapid publication on acceptance

- support for research data, including large and complex data types

- gold Open Access which fosters wider collaboration and increased citations

- maximum visibility for your research: over $100 \mathrm{M}$ website views per year

At $\mathrm{BMC}$, research is always in progress.

Learn more biomedcentral.com/submissions 